\title{
ON THE EULER SUMMABILTTY OF A CLASS OF DIRICHLET SERIES
}

\author{
V. F. CowLING'
}

(Keseived June 28, 1955)

The object of this paper is to study the Euler-Knopp summability, denoted $E(r)$, of Dirichlet series $\sum_{n=0}^{\infty} a_{n} e^{-r_{n} z}$ for the case in which $r_{n}=(n+1)^{a}$ for $0<\alpha<1$. If $\alpha=1$ we have a Taylor series under the substitution $\xi=e^{-z}$ and this problem has been studied extensibely by Agnew [1], Knopp [3] and many others. N. Obreschkoff [4] has made a study of this problem in the case $r_{n}=\log (n+1)$. The Euler-Knopp series-to-series transformation, carries the series $\sum_{n=0}^{\infty} a_{n}$ into the series $\sum_{n=0}^{\infty} A_{n}$ where $A_{n}$ is defined by

$$
A_{n}=\sum_{k=0}^{n}\left(\begin{array}{l}
n \\
k
\end{array}\right) r^{k+1}(1-r)^{n-k} a_{k,}, \quad 0<r<1
$$

for $n=0,1,2, \ldots$. This transformation carries convergent series into series that converge to the same sum.

The following theorem is proved.

TheOREM. Let $A_{n}=\sum_{k=0}^{n}\left(\begin{array}{l}n \\ k\end{array}\right) r^{k+1}(1-r)^{n-k} a_{k} e^{-\left(k^{2}+1\right)^{\alpha} z_{0}}$. Suppose $\sum_{p=0}^{\infty} A_{p} r^{-p}$ converges for some $0<r_{0}<1$. Then $\sum_{n=0}^{\infty} a_{n} e^{-(n+1)^{\alpha_{z}}}$ is absolutely summable $[(r)$. for $r>r_{0}$ for those $z$ satisfying the inequality $\pi(1-\alpha) / 2>\left|\operatorname{Arg}\left(z-z_{0}\right)\right|$.

Proof. As is well known we can solve equations (1) for the $a$ : to obtain.

$$
a_{k}=\sum_{p=0}^{k}\left(\begin{array}{l}
k \\
p
\end{array}\right) r^{-p-1}\left(1-\frac{1}{r}\right)^{k-p} A_{p}
$$

for $k=0,1,2, \ldots$.

As applied to the series $\sum_{n=0}^{\infty} a_{n} e^{-(n+1)^{\alpha}} z_{0}$ this implies that

$$
\left.a_{i:} e^{-(k+1)^{\alpha} z_{0}}=\sum_{p=0}^{k} \begin{array}{l}
k \\
p
\end{array}\right) r^{-p-1}\left(1-\frac{1}{r}\right)^{k-p} A_{p}
$$

and the series whose convergence is to be established can be written in the form

1) This research was supported in part by a grant from the National Science Foundation. 


$$
\sum_{n=0}^{\infty}(1-r)^{n} \sum_{k=0}^{n}\left(\begin{array}{l}
n \\
k
\end{array}\right) r^{k+1}(1-r)^{-k} a_{k:} e^{-(k+1)^{\alpha_{z}}} \equiv \sum_{n=0}^{\infty}(1-r)^{n} B_{n}
$$

where

(4) $\quad B_{n}=\sum_{k=0}^{n}\left(\begin{array}{l}n \\ k\end{array}\right) r^{k+1}(1-r)^{-k} e^{-(k+1)^{\alpha}\left(z-z_{0}\right)} \sum_{p=0}^{k}\left(\begin{array}{l}k \\ p\end{array}\right) r^{-p-1}\left(1-\frac{1}{r}\right)^{k-p} A_{p}$.

Formally reversing the lorder of summation in the right member of (4) yields the result

(5) $B_{n}=\sum_{p=0}^{n} r^{-p-1}\left(1-\frac{1}{r}\right)^{-p} A_{p} \sum_{k=p}^{m}\left(\begin{array}{l}n \\ k\end{array}\right)\left(\begin{array}{l}k \\ p\end{array}\right)(1-r)^{-k}\left(1-\frac{1}{r}\right)^{k} e^{-(k+1)^{\alpha}\left(z-z_{0}\right)}$

Now $\left(\begin{array}{l}n \\ k\end{array}\right)\left(\begin{array}{l}k \\ p\end{array}\right)=\left(\begin{array}{l}n \\ p\end{array}\right)\left(\begin{array}{c}n-p \\ k\end{array}\right)$. By use of the Mellin transform [2, p. 116] we may show that if $s=\sigma+i t$

$$
e^{-(k+p+1)^{\alpha}\left(z-z_{0}\right)}=\frac{1}{2 \pi i} \int_{\sigma-i \infty}^{\sigma+i \infty}\left(z-z_{0}\right)^{-s} \frac{\Gamma(s)}{(k+p+1)^{\alpha s}} d s
$$

if $\sigma>0$ and $\Re\left(z-z_{0}\right)>0$, the latter inequality holding by our hypothesis. Also by the theory of the Gamma function it follows that

$$
(k+p+1)^{-\alpha s}=\frac{1}{\Gamma(\alpha s)} \int_{0}^{\infty} \xi^{\alpha s-1} e^{-(k+p+1) \xi} d \xi
$$

valid if $\Re(s)>0$. Combining there two results we have

$$
e^{-(k+p+1)^{\alpha}\left(z-z_{0}\right)}=\frac{1}{2 \pi i} \int_{\sigma-i \infty}^{\sigma+i \infty}\left(z-z_{0}\right)^{-s} \frac{\dot{\Gamma}(s)}{\Gamma(\alpha s)} \int_{0}^{\infty} \xi^{\alpha s-1} e^{-(l i+p+1)} d \xi d s
$$

valid if $\sigma>0$ and $\mathfrak{R}(z)>\Re\left(z_{0}\right)$. Thus after some simplifications equation (5) may be rewritten in the form

$$
\begin{aligned}
B_{n}=\sum_{p=0}^{n} \mathrm{~A}_{p}\left(\begin{array}{l}
n \\
p
\end{array}\right)(1-r)^{-p} \frac{1}{2 \pi i} \int_{\sigma-i \infty}^{\sigma+i \infty}\left(z-z_{0}\right)^{-s}\left(\begin{array}{c}
\Gamma(s) \\
\Gamma(\alpha s)
\end{array}\right) \\
\\
\int_{0}^{\infty} \xi^{\alpha s-1} e^{-(p+1) \xi}\left(1-e^{-\xi}\right)^{n-p} d \xi d s
\end{aligned}
$$

Let us now consider the series

$$
\sum_{n=0}^{\infty}(1-r)^{n} B_{n}
$$

Under the summation sign in (6), let $A_{p}$ be replaced by $\left|A_{p}\right|$ and let the integrands be replaced by their absolute values. Let the resulting expression be substituted for $B_{n}$ in the series (7); and let the order of summation in the result of this substitution be reversed. The resulting series omitting the factor $(2 \pi)^{-1}$ then is of the form 


$$
\begin{aligned}
\sum_{p=0}^{\infty}\left|A_{p}\right| \sum_{n=p}^{\infty}\left(\begin{array}{l}
n \\
p
\end{array}\right)(1-r)^{n-p} & \int_{\sigma-i \infty}^{\sigma+i \infty}\left|\left(z-z_{0}\right)^{-s}\right|\left|\frac{\Gamma(s)}{\Gamma(\alpha s)}\right| \\
& \cdot \int_{0}^{\infty} \xi^{\alpha \sigma-1} e^{-(p+1) \xi\left(1-e^{-\xi}\right)^{m-p} d \xi d t .}
\end{aligned}
$$

Since $(1-r)\left(1-e^{-\xi}\right)<1$ for $0<r<1$ and all real $\xi$ it follows that the order of the second summation sign and the integral may be reversed to obtain

$$
\sum_{p=0}^{\infty}\left|A_{p}\right| \int_{\sigma-i \infty}^{\sigma+i \infty}\left|\left(z-z_{0}\right)^{-s}\right|\left|\frac{\Gamma(s)}{\Gamma(\alpha s)}\right| \int_{0}^{\infty} \xi^{\alpha \sigma-1}\left[1+r\left(e^{\xi}-1\right)\right]^{-p-1} d \xi d t .
$$

If now the series (8) converges this will imply the absolute convergence of the series (7) and complete the proof of the theorem. To this end we note that a simple calculation shows

$$
\int_{0}^{\infty} \xi^{\alpha \sigma-1}\left[1+r\left(e^{\xi}-1\right)\right]^{-p-1} d \xi=O\left(r^{-(p+1)}\right)
$$

as $p$ becomes infinite. Also we recall that $[2, p .116]$

$$
s=\sigma+i t
$$

$$
|\Gamma(s)|=e^{-(\pi / 2)|t|}|t|^{\sigma-1 / 2}(\sqrt{2 \pi}+O(|t|)) \text {, }
$$

If this is taken into account it is easily seen that the first integral in the expression (8) converges for those $z$ for which

$$
\pi(1-\alpha) / 2>\left|\operatorname{Arg}\left(z-z_{0}\right)\right| \text {. }
$$

Therefore the series (8) will converge and the series (7) converge absolutely for those $z$ satisfying (9) if

$$
\sum_{p=0}^{\infty}\left|A_{p}\right| \boldsymbol{r}^{-p}
$$

converges. Since $\sum_{p=0}^{\infty} A_{p} r^{-p}$ converges by hypothesis for some $0<r_{0}<1$ this completes the proof of the theorem.

\section{BiBLIOGRAPHY}

[1] R. P. Agnew, Euler Transformations, Amer. Journ. Math, 66 (1944), 313-338.

[2] G. Doetsch, Theorie und Anwendung der Laplace-Transformation, Dover Publications (1943).

[3] K. KNOPP, Über das Eulersche Summierungsverfahren, Math. Zeit., 15 (1922), 226253.

[4] N. OBRESCHKOFF, Anwendung der Eulerschen Reihentransformation, Sitzungsberichte der Preuszeschen Akademie der Wissenschaften, (1938), 267-302.

Department of MAthematics And Astronomy, University of Kentucky. 